\title{
Todd's Paralysis
}

National Institute of Neurological Disorders and Stroke (NINDS)

\section{Source}

National Institute of Neurological Disorders and Stroke (NINDS). Todd's Paralysis

Information Page.

Todd's paralysis is a neurological condition experienced by individuals with epilepsy, in which a seizure is followed by a brief period of temporary paralysis. The paralysis may be partial or complete but usually occurs on just one side of the body. The paralysis can last from half an hour to 36 hours, with an average of 15 hours, at which point it resolves completely. Todd's paralysis may also affect speech and vision. Scientists don't know what causes Todd's paralysis. Current theories propose biological processes in the brain that involve a slow down in either the energy output of neurons or in the motor centers of the brain. It is important to distinguish Todd's paralysis from a stroke, which it can resemble, because a stroke requires completely different treatment. 